\title{
Illustrations and Figures
}

1.1 "High Park Forest School class at work," 192632

1.2 "Forest School - Board of Education," 191333

1.3 "Orde Street Open Air School," 191934

1.4 "Forest School - Board of Education - High Park," 191335

2.1 "Special classes for mental defectives," 192461

2.2 "Mental hygiene in primary schools," 192462

2.3 Eric Kent Clarke, ca. 191063

2.4 "Auxiliary class mental test," ca. 193068

2.5 "Boy caning chair, auxiliary class," ca. 192081

3.1 "Edith [L.] Groves school," 192997

3.2 "Junior vocational school, pyramid by gym class," 193098

3.3 Junior vocational school, auto shop, 1930110

3.4 "All the trimmings," 1939111

3.5 Average daily attendance (ADA), selected Toronto secondary schools, 1925-35 121

3.6 Junior vocational school enrolments, 1926-40 123

4.1 "Vocational school opening, Mrs Groves and Premier Ferguson," 1929133

4.2 "Transportation, crippled scholars," 1926134

4.3 Children on ramp, Wellesley Public School, ca. 1926-30 147

4.4 Children at Christmas event, Wellesley Public School, 1927148

5.1 Toronto Department of Mental Hygiene, Selected Recommendations, 1935-45 168

A.1 3" $\times$ 5" Office record card (ORC) 215

A.2 5"x 8" Admission-discharge-promotion (ADP) card 216

A.3 Psychiatric examination report (PER) 217 


\section{x Illustrations}

A.4 Hester How Public School, 1920s 218

A.5 Coleman Ave. Public School, 1910220

A.6 Duke of York Public School, 1939221 